\title{
LA PENISOLA BALCANICA TRA ORIENTE E OCCIDENTE DEL SECOLO XIII
}

\author{
Momcillo Spremic
}

Quando nel 395, dopo la morte dell'imperatore Teodosio I, I'Impero romano fu diviso in due parti, il confine tra esse passava nel mezzo dell'Illiria, dunque attraverso la penisola Balcanica. Esso si stendeva dalla costa adriatica orientale, seguendo pressappoco il corso del fiume Drina, verso il nord fino a Belgrado. Le provincie Moesia Superior, Dardania e Prevalis appartennero all'Oriente, mentre la Pannonia e la Dalmazia rimasero in Occidente. Le conseguenze di quella partizione si fecero sentire nel corso di tutto il Medioevo, quando i Balcani vennero abitati da popoli di origine etnica eterogenea: Greci, Slavi, che vi si installarono nei secoli vi e VII, Albanesi e anche da discendenti degli antichi romani. Questo conglomerato etnico oscillo durante i lunghi secoli del Medioevo tra Oriente e Occidente tra la chiesa ortodossa e quella cattolica, tra la scrittura greca e quella latina. E'particolarmente interessante vedere che cosa ne successe nel secolo XIII. 
La storia della penisola Balcanica del secolo XuI è assai complessa. Vi si trovavano alcune unità statali che erano in loua tra di loro per l'egemonia e nello stesso tempo lottavano contro le forze straniere. Questi stati erano l'Impero bizantino, la Serbia e la Bulgaria. Nessuno di essi era riuscito ad ottenere il predominio su questa regione, grandemente anche perchè la storia della penisola balcanica nel secolo XIII era basata sulle conseguenze della quarta crociata. Crollato I'Impero bizantino nel 1204, venne fondato l'Impero latino con la sede a Costantinopoli. Altre fondazioni latine erano il Regno di Salonícco, il Ducato di Atene, il Principato di Acaia. I Greci fondarono l'Impero di Nicea nell'Asia minore, ma anche il Despotato d'Epiro nella parte occidentale della penisola balcanica. Tuttavia il maggiore profitto della quarta crociata trassero i veneziani. Essi occuparono degli importanti strategiei e porti lungo la costa balcanica, quasi dalla stessa Venezia fino a Constantinopoli ${ }^{1}$.

Intanto la situazione creatasi nel 1204 non durò a lungo. Dal 1216 l'Impero latino andava decadendo e nel 1224 il despota dell'Epiro greca Teodoro Angelo (1215-1230) occupò il regno di Salonicco.

Il destino degli stati slavi meridionali era determinato dai rapporti esistenti tra il mondo orientale, ossia bizantino e quello occidentale. Il crollo dell'Impero bizantino nel 1204 influi direttamente sulla decisione del re bulgaro Kalojan (1197-1207) di rivolgersi all'Occidente e di cominciare a chiedere la corona a Roma. Le trattative ebbero un buon esito e già l'8 novembre del 1204 egli venne solennemente

1 G. Ostrogorski, Isterija Vizantije (La storia di Bisanzio), Beograd 1969 pp., 396-400. Sulla dominazione dei latini in Oriente dopo la quarta crociata: E. GERLAND, Geschichte des lateinischen Kaiserreiches von Konstantinopel, I, Geschichte der Kaiser Balduin I und Heinrich 1204. 1216, Hamburg v. d. Hohe 1905; W. MILLer, The Latins in the Levant. A History of Frankich Greece (1204-1566), London 1908; W. MILLER, Essayson the Latin Orient, Cambridge 1921; J. LoNGNON, L'Empire latin de Costantinople et la principauté de Morée, Paris 1949; R. WOLFF, "The Latin Empre of Constantinopel, 1204-1261.» A History of the Crasades 11 (1189-1311), Madison 1969. 
incoronato a Trnovo da parte del legato papale, cardinale Leone $^{2}$. Il suo esempio segui il regnante della Serbia Stefano Nemanjić (1196-1223), che portava il titolo di Grande Zupan e aspirava a quello di re. Egli chiese esplicitamente a Roma il diadema regale (regium diadema), e il papa incaricò Giovanni, l'episcopo della città di Albano in Lazio, all'inizio del secolo XIII, di incoronarlo. Però questo non avvenne in quell'occasione per l'opposizione del re ungaro. Intanto l'affare non andò perduto, ma la cerimonia venne solamente rimandata. Avendo ripudiata la moglie, che era una principessa bizantina, Stefanò sposò la nipote del celebre doge veneziano Enrico Dandolo la quale cosa gli facilitò a ottenere a Roma la meta anelata. E già nel 1217 Stepbanus, dominus Servis sive Rasie, fu incoronato nel proprio paese da parte del legato papale. Cosi la Serbia divenne un regno sotto la dinastia dei Nemanjidi $^{3}$.

Quando nel 1230 l'imperatore bulgaro Ivan Asen II (1218-1241) sconfisse nella battaglia di Klokotnica sul fiume Marica l'esercito del despota d'Epiro Teodoro Angelo, il predominio per un certo tempo lo ebbe la Bulgaria. Ma non per lungo, solo fino al 1241 quando Ivan Asen II mori. Proprio in quell'anno furono scossi tutti i popoli dei Balcani. Durante il Medioevo essi furono più volte vittime di diferenti conquistatori asiatiei che facevano irruzioni in Europa. Nell'alto Medioevo lo furono unni, avari, poi ungari e propio tra il 1240 e 1241 i mongoli. Dopo la morte di Gengis-Kan, una parte di essi irruppe in Russia e poi in Pologna, Boemia, Moravia e Ungheria per arrivare fino alla stessa Dalmazia. Distruggendo le citta e uccidendo la popolazione che ci trovava, il loro capo Kajdan tornò attraverso la Bosnia, la Serbia e la Bulgaria sul basso Danubio. Quest'impresa mongola, violenta come una procella, lasciò gravi conseguenze nei paesi balcanici, i quali già erano in conflitti reciproci continuamente.

Di queste controversie nei Balcani approfittò abilmente l'imperatore di Nicea Giovanni III Vatae (1222-1254), che prese territori considerevoli alla Bulgaria indebolita, ai

${ }^{2}$ V. ZLATARSKI, Istorija na Blgarskata drrzava prez srednite vekove (La storia dello Stato bulgaro nel Medioevo), III, Sofija 1940, pp. 149-211. 169.

K. JIRECEK, Istorija Srba (La stoira dei Serbi), I, Beograd 1952, pp. 168 - 
despoti d'Epiro per occupare nel 1246 Salonicco. Con ciò egli fece un passo decisivo che lo avvicinò all'occupazione di Costantinopoli e alla restaurazione dell'Impero bizantino. Il se serbo Stefano Uros I (1243-1276) ebbe rapporti d'amicizia con il sempre più potente Impero di Nicea, sebbene egli esitasse tra l'Epiro, gli ungheresi e il re di Napoli. Tra questi ultimi, Federico II Hohenstaufen (1212-1250), senza dubbio il più grande regnante europeo del secolo XIII, era amico dei greci, mentre suo figlio Manfredi (1250-1266) riprese l'antica politica antibizantina dei re normanni. Nel $1258 \mathrm{egli}$ occupò Corfù, Durazzo, Valona e le città d'Epiro. Dalla sua parte si misero il despota d'Epiro, i signori latini in Grecia e il re serbo Stefano Uros I. Tuttavia, nell'autunno del 1259 nella pianura Pelagonia l'esercito eterogeneo degli alleati venne sconfitto dalle truppe bizantine dell'imperatore di Nicea $^{4}$. Questo fatto, tra l'altro, aiutò Michele VIII Paleologo (1259-1282) a occupare Costantinopoli e quindi restaurare, dopo sei decenni, l'Impero bizantino unificatos.

L'ostilità verso l'Impero di Costantinopoli venne espressa dall'Italia meridionale ancora più esplicitamente quando sul trono del Regno delle due Sicilie era salito Carlo I d'Angiò (1266-1285). Da un re cattolico dichiarato egli iniziò la lotta contro la Bisanzio "scismatica». Cominciò ad aiutare i signori latini del Peloponneso e nel 1272 occupò Durazzo, la "porta di Bisanzio", e città vicine. Quindi fondò il suo regnum albanie. In una tale situazione i regnanti degli stati slavi meridionali, della Serbia e della Bulgaria, si misero dalla parte del nuovo re di Napoli. La posizione dell'imperatore bizantino si fece gravissima all'inizio del nono decennio del secolo XIII quando $i$ veneziani si misero dalla parte di Carlo I d'Angiò e la Curia romana venne sotto il suo controllo. All'ultimo momento le cose cambiarono. Michele VIII, per li tramite dei genovesi, prese contatto con il re aragonese Pietro III. L'imperatore bizantino aveva messo a disposizione dell'Aragonese i mezzi per la costruzione della flotta, e nello stesso tempo agenti bizantini, provveduti di

4 D. Geanaxoplos, Greco-Latin Relations on the Eve of the Byzantine Restauration: the Battle of Pelagonia, Dumbarton Oaks Papers, 7 (1953), pp. 99-141.

s Sulla restaurazione dell'Impero bizantino sotto Michele VIII Pleologo: C. Chapman, Michel Paléalogue, restaurateur de l'Empire byzantin, Paris 1926. 
denaro, andavano istigando la popolazione siciliana contro il potere angioino. Il 31 marzo del 1282 coi Vespri Siciliani di Palermo a Carlo I d'Angiò fu inferito un colpo da cui egli non potè più riprendersi. Egli perse la Sicilia e quindi come signore solo dell'Italia meridionale non era più un avversario serio dell'imperatore di Costantinopoli.

La posizione degli altri regnanti della penisola balcanica venne determinata dalle lotte tra gli angioini e Bisanzio. Questo riguarda i signori della Tessaglia, la quale nel frattempo si era appartata dell'Epiro ed era continuata ad esistere come uno stato indipendente. Poi, ciò riguarda anche l'imperatore bulgaro $\mathrm{e}$ in modo particolare il re serbo Stefano Milutin (1282-1321). Ignorando i Vespri Siciliani, quest'ultimo prese nel 1282 Skopeje ed altre città macedoni ai bizantini ${ }^{6}$. Proprio a quel tempo comincià l'espansione dello stato serbo verso il sud a spese di Bisanzio. Solo aleuni decenni più tardi la Serbia si sarebbe estesa fino al Golfo di Corinto e il suo re si sarebbe proclamato imperatore. Dall'altra parte, a Oriente, l'Impero bizantino cominciò ad essere represso dai turchi ottomani e quindi alla fine del secolo XIII e all'inizio del secolo XIV esso diveine uno stato di secondo ordine.

Cosi il contatto fra Oriente e Occidente su un piano politico venne personificato dalle lotte tra Bisanzio e l'Occidente. L'influsso occidentale proveniva dall'Italia ma anche dall'Ungheria. Occorre menzionare che $i$ re ungheresei avevano in possesso la Croazia e saltuariamente anche le regioni settentrionali della Serbia e della Bulgaria, i cui re erano considerati i loro vassalli. Gli altri stati europei del secolo XIII non ebbero un influsso diretto sugli eventi politici nei Balcani. La Russia era frantumata in molti principati e intorno all'anno 1240 cadde sotto il giogo tartaro. L'influsso dell'Impero germanico non si sentiva. Del resto, nella seconda metà del secolo XIII esso si trovò in un interregnum e quando Rodolfo d'Asburgo (1273-1291) fue eletto al trono, egli ayeva molte preoccupazioni nel proprio impero. Le relazioni con la lontana penisola iberica si limitavano a pochi contatti tra il re bizantino e il re

${ }^{6}$ Sul re Milutin: L. Mavromatis, La fondation de l'Empire serbe. Le kralj Milutin, Thessaloniki, 1978, pp. 15-84. 
aragonese. In verità, un pò più tardi questi contatti si fecero più intensi. Sono assai note le attività della Compagnia catalana nell'Impero bizantino del secolo XIV, però si sa pochissimo sui mercenari spagnoli che nel 1330 , al servizio del re serbo, parteciparono alla battaglia di Velbuzd contro i bulgari $^{7}$. Qualche pellegrino francese o inglese passava nel secolo XIII vicino alle coste balcaniche sulla strada verso i luoghi sacri cristiani in Oriente, però né la Francia né l'Inghilterra influirono sugli avvenimenti balcanici di quel tempo.

\section{II}

Anche nell'economia dei paesi balcanici vennero a contatto l'Oriente e l'Occidente.

Come altrove nel Medioevo la popolazione si occupava per lo più di agricoltura. Benchè nel secolo XIII i latini avessero occupato vasti territori bizantini, essi non fecero cambiare molto la produzione agraria. I contadini continuarono a vivere e a lavorare alla loro maniera tradizionale, servissero il padrone greco o latino. Quanto alla popolazione slava, essa si trovò sotto il dominio bizantino e quindi iniziò la sua vita da agricoltori sotto l'influsso degli esempi bizantini. L'adozione delle leggi agrarie bizantine nonchè la rispettiva terminologia d'origine greca ne sono testimonio ${ }^{8}$. L'influsso occidentale sull'agricoltura fu minore e si sentiva

7 M. DiNIC, Spanski najamniei u srpskoj sluzbi (Mercenari spagnoli al servizio dei Serbi), Zbornik radova Vizantoloskog instituta u Beogradu, 6, 1960, pp. $15-28$.

${ }^{3}$ M. BLsgojevic, Zemljoradnja u srednjovekovnoj Srbiji (L'agricoltura nella Serbia medievale), Beograd 1973, p. 60. Cfr. anche M. Vlajinac, Die agrarrechtlicben Verbältnisse des Mittelalterschen Serbiens, Jena 1903. Sulla situazione agraria nell'Impero bizantino: G. OsTROGORSKY, "Agrarian Conditions in the Byzantine Empire in the Middle Agesn, Cambridge Economic History, 1,1941 , pp. 205-234; pp. 774-779. 
soprattutto nelle zone costiere 9 . Una grande parte della popolazione organizzata in comunità tipicamente balcaniche si occupava dell'allevamento del bestiame. Le corporazioni d'artigiani di tipo occidentale non erano conosciute che nelle città marittime.

Una particolarità del secolo XIII fu lo sviluppo violento della produzione mineraria. In Serbia, in Bosnia e in Bulgaria furono i tedeschi, conosciuti nei paesi balcaniei come sassoni, a farla progredire. Dalle fonti disponibili risulta esplicitamente che era stato il re serbo Uros I a farli venire. Quindi l'influsso dell'Occidente su quest'attività economica fu decisivo. Coll'andar dell tempo i sassoni fecero apprendere la tecnica mineraria agli indigeni. Essi si slavizzarono poco a poco, sebbene una buona parte della terminologia mineraria rimase quella d'origine tedesca fino alla fine del Medioevo. Nelle miniere balcaniche vennero estratti ferro, rame, piombo e soprattutto argento. Questo ultimo metallo veniva esportato in varie parti e specialmente sulla costa adriatica orientale attraverso cui arrivava nella vasta regione del Mediterraneo. Nel tardo Medioevo l'Europa era golosa dei metalli preziosi e particolarmente d'argento. Dalle ricerche scientifiche recenti risulta che una buona parte dei fabbisogni, specialmente quelli del bacino economico mediterraneo, furono coperti proprio coll'argento balcanico. Il materiale del ricco Archivio di Ragusa, serbato in verità solo dal 1280 in poi, ci fa constatare come anche dei mercanti catalani acquistassero questo argento ${ }^{10}$.

Fino alla quarta crociata gli abili mercanti greci dominarono la maggior parte della penisola balcanica. Pertanto, nel 1204 , con il crollo di Bisanzio, il loro ruolo fu preso dai

9 I. Bozıć, "Le système foncier en "Albanie venittienne" au XV' siècle", Bollettino dell'Istituto di Storia della Società e dello Stato veneziano, V-VI (19631964, pp. 65-140. Sull'agricoltura dell' Occidente europeo nel Medioevo: G. DUBY, L'economie rurale et la vie des campagnes dans /"Occidente médiéval, I-IV, Paris 1962.

10 Sulla produzione mineraria nella Serbia e Bosnia medievale: M. Dinić, Za istoriju rudarstva u srednjevekovnoj Srbiji i Bosni (Per una storia dell'industria mineraria nella Serbia e Bosnia Medievale), I, Beograd 1955; II, Beograd 1962; K. JIRECEK, "Die handelsstrassen und Bergwerke von Serbien und Bosnien während des Mittelalters", Abbandlungen der kónigl. bähm. Geselschaft der Wissenschaften, VI Folge, 10 Band, Prag 1879. 
veneziani ${ }^{11}$. Per far neutralizzare la loro potenza, l'imperatore Michele VIII Paleologo prese contatti con i loro avversari, i genovesi. Coll'accordo del 1261 egli concesse loro molti privilegi commerciali e in questo modo furono gettate le basi della grandezza di Genova in Oriente ${ }^{12}$. Benchè Venezia e Genova lottassero tra di loro durante la seconda metà del secolo XIII, esse andarono sempre più consolidando le loro posizioni a spese di Bisanzio, che alla fine divenne prigioniera delle repubbliche mercantili italiane.

Dall'attività commerciale degli italiani non furono compresi soltanto i paesi greci, ma anche quelli slavi della penisola balcanica. Specialmente attivi erano i veneziani. Essi acquistavano argento, e vendevano prodotti d'artigianato, merce di lusso e tessuti. La loro sempre più voluminosa attività si rifletteva anche sulla circolazione della moneta. L'antica bene conosciuta moneta bizantina venne oppressa dai ducati veneziani, che erano coniati proprio nel secolo XIII. Essi diventarono relativamente presto il mezzo di scambio sul mercato del Mediterraneo e quindi anche nei paesi slavi dei Balcani. La prima moneta serba fu coniata sul modello di quella veneziana. Venezia protestò più volte a causa di cio, e Dante Alighieri rese immortale il re serbo Stefano Milutin menzionandolo come inmitatore del conio veneziano nella Divina Commedia.

Un ruolo di particolare importanza nello scambio commerciale tra la penisola balcanica e l'Occidente ebbe Ragusa. Questa città-stato situata sulla costa adriatica orientale stipulò nel 1201 e 1211 contratti di reciproci privilegi con Bari, Monopoli, Termoli, Molfetta e Bisceglie e più tardi con Arcona ed altri centri della sponda opposta ${ }^{13}$. Questo rese possibile ai sudditi di Ragusa di svilupparci una estesa attività commerciale. Dall'altra parte essi penetravano profondamente nel retroterra balcanico e acquistavano prodotti

11 Sulla politica veneziana in Oriente: F. THIRIET, La Romanie vénitienne an Moyen age. Le développement et lexploitation du domaine colonial vénetien XII-XV sticcle, Paris 1959.

${ }^{12}$ W. HEYD, Histoire du commerce du Levant an Moyen age, Leipzig 1921,427; G. Bratianu, Recherches sur le commerce génois dans la Mer Noire au XIIT siécle, Paris 1929 , p. 81.

13 J. RADONIC, Acta et diplomata Ragusina, tomus I, fasc. 1. Belgradi 1934, pp. 14-18. 
degli allevatori del bestiame e metalli, che poi vendevano in Italia e in tutta la regione del Mediterraneo. Cosi essi furono veri intermediari tra la vasta regione balcanica e il ricco bacino mediterraneo. Il loro commercio era basato sulle forme organizzative corrispondenti a quelle esistenti nell'Occidente europeo ${ }^{14}$.

Il sopraesposto fa vedere come nel secolo XIII i paesi balcanici furono grandemente compresi dall'attività dei mercanti appartenentí al mondo occidentale. Anzi, si può dire deliberatamente che a quel tempo l'influsso dell'Occidente sui popoli balcanici venne esercitato proprio nel campo economico.

\section{III}

È una cosa particolarmente interessante esaminare la situazione ecclesiastica sulla penisola balcanica nel secolo XIII. La maggior parte di questa regione apparteneva alla chiesa orientale, ortodossa, e la minore a quella occidentale, cattolica. Sul piano teologico i cristiani orientali e quelli occidentali si separarono intorno al famoso problema di Filioque. I primi sostenevano che lo Spirito Santo promana solo dal Padre, mentre gli altri consideravano che lo Spirito Santo promana dal Padre e dal Figlio. A ciò si aggiunse un contrasto istituzionale, siccome il patriarca di Constantinopoli rifiutò di riconoscere il primato del papa. Il dissenso si trasformò col tempo in odio. I latini rimproveravano i greci di essere sofisticati, codardi ed ingannatori e soprattuto li

14 Sul commercio ragusino nel retroterra balcanico: M. DinIC, «Dubrovacka srednjevekovna karavanska trgovina" (Il commercio carovaniero ragusino nel Medioevo), Jugoslovenski istorijski casopis, 3, 1937, pp. 119-146. Sul credito commerciale: I. VoJE, Kreditna trfovina u srednjovekovnom Dubrovniku (II credito commerciale nella Ragusa medievale), Sarajevo 1976, pp. 87-343. Sui rapporti economici con i paesi aragonesi: M. SPREMIC, Dubrounik i Aragaonci (Ragusa e gli Aragoneisi), Beograd 1971, pp. 29-181. 
odiavano perchè erano riechi. All'odio dei latini i greci rispondevano col disprezzo degli occidentali come gente barbara.

Quando all'inizio del secolo XIII i crociati partivano per l'Oriente, il clero occidentale giustificava limpresa contro la scismatica Bisanzio, accentuando che quella era persino un opera pia. Proprio quello fu il periodo della maggiore potenza del papato alla testa del quale allora si trovava Innocenzo III (1198-1216). Il suo potere si sentiva non soltanto nell'Europa occidentale bensi anche in quella orientale fino alla lontana Armenia. Innocenzo III era promotore della quarta crociata, con cui fu inferito un gran colpo non soltanto allo stato bizantino ma anche alla chiesa cristiana d'Oriente. Dal 1204 fino al 1216, dalla caduta di Costantinopoli fino alla fine del suo pontificato, egli riusci a dominare la chiesa greca. Intanto no lo poterono fare più i suoi successori il cui pontificato coincise con la decadenza dell'Impero latino.

Nella stessa chiesa greca dopo l'anno $1204 \mathrm{i}$ diritti del patriarca di Costantinopoli vennero assunti dal patriarca di Nicea. Già nella prima metà del secolo XIII il papato iniziò le trattative coll'imperatore di Nicea per l'unione delle due chiese cristiane. L'imperatore Giovanni III Vatatze manteneva contemporaneamente rapporti con l'imperatore Federico II Hohenstaufen, l'avversario del papa e con la Curia romana. E interessante dire che Federico II, conosciuto per la sua tolleranza religiosa, esprimesse sincere simpatie per i greci «i quali erano calunniati sfrontatamnete e chiamati eretici da parte del cosiddetto pastore supremo (ossia papa), sebbene da loro derivasse la religione cristiana che si diffondeva fino agli estremi confini del mondo ${ }^{15}$. Nelle trattative con Roma Giovanni III Vatatze era disposto a sacrificare l'indipendenza della propria chiesa, a condizione che il papa rinunciasse all'Impero latino e in tal modo rendesse possibile il ritorno dell'imperatore a Costantinopoli ${ }^{16}$. In quell'Occasione però non fu raggiunto accordo ed il suo successore

is G. Ostrogorski, op. cit., pp. 413.

16 W. NoRden, Das Papstum und Byzanz. Die Tretanung der beiden Mãcbte und das Problem ihrer Wiedervereinigung bis zum Untergange des Byzantiniscben Reiches, Berlin 1903, p. 359. 
Teodoro II Lascaris (1254-1258) inizio una politica ecclesiastica del tutto contraria. Egli non volle nemmeno saperne della sottomissione della chiesa greca al papa. Soprattutto i monaci del Monte Atos erano avversari del unione. All'inizio del secolo XIII essi venivano derubati dai latini i quali li costringevano con la forza ad accettare l'unione ${ }^{17}$.

Il papato accolse di malavoglia la restaurazione di Bisanzio del 1261 non potendo rassegnarsi al fatto che Costantinopoli fosse perduta per la chiesa romana. Tuttavia, quando Carlo I d'Angiò si era avviato per una politica antibizantina, l'imperatore Michele VIII Paleologo cominciò ad offrire al papa l'unione. Avendo ottenuto da Gregorio X (1271-1276) la garanzia di sicurezza da parte delle forze cattoliche, l'accordo fu presto concluso. Quindi al Concilio di Lione il 6 giugno del 1274 la delegazione bizantina composta da alti dignitari di Stato e di Chiesa firmo l'Unione riconoscendo non solo il primato della chiesa cattolica ma anche il dogma di Roma ${ }^{18}$.

Con la proclamazione dell'unione Michele VIII fece migliorare la sua posizione nel campo della politica estera, però s'imbattè in una grande opposizione da parte dei suoi sudditi. Il popolo bizantino e la maggiore parte del clero bizantino non volle saperne dell'unione. Quello stesso popolo da secoli era stato educato a considerare la religione ortodossa una cosa sacrosanta ed a odiare $i$ latini profondamente. Per questo essi si ribellarono contro l'imperatore che aveva tradito la religione degli antenati. Tutto l'impero fu diviso in due campi. In una tale situazione l'imperatore non potè fare altro che mettere in atto l'unione a ferro e fuoco e di far perseguitare atrocemente i numerosi avversari. Però in tal modo l'ùnione non potè essere messa in atto. Anzi, dopo breve tempo essa venne respinta dalla stessa Roma.

Venuto sotto l'influsso del bellicoso Carlo I d'Angiò, il papa Martino IV (1281-1285) scomunicò l'imperatore Mi-

17. M. Zivojinovic, "Sveta Gora u doba Latinskog carstva» (Athos al tempo dell'Impero latino) Zbornik radova Vizantoloskog instituta u Beogradu, 17 , 1976 , pp. 77-91. Per un breve periodo, verso la fine del pontificato di Innocenzo III, anche Athos riconobbe l'autorità suprema del papa. G. HoFmanN, "Rom und Athosklöster", Orientalia cbristiana VIII, 1, 1926, pp. 7 -

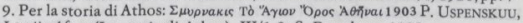
Istorija Afona (La storia di Athos), III/1-2, S. Peterburg 1892.

is G. Ostrogorski, op. cit., pp. 430-434. 
chele VIII come scismatico e vietò aglire cattolici di mantenere rapporti con lui ${ }^{19}$. Cosi l'unione proclamata a Lione ebbe un totale insuccesso. Il nuovo imperatore bizantino Andronico II Paleologo (1282-1328) la respinse solennemente e si avviò per una politica completamente ortodossa ${ }^{20}$.

I rapporti tra l'Impero bizantino e l'Occidente nel campo ecclesiastico influirono sull'oscillazione dei paesi slavi dei Balcani tra Roma e Costantinopoli, quali centri delle due chiese cristiane. Le regione della Slovenia e della Croazia erano inquadrate nel secolo XIII, come anche prima e dopo quel periodo, nella chiesa occidentale. Intanto, in Bosnia esisteva una particolare chiesa eretica considdetta patarina. Non penetrando in questa sede nel suo insegnamento, menzioneremo che la sua caratteristica essenziale consisteva nel dualismo. Nel mondo lottano sempre due principi: quello spirituale e quello materiale, personificati da due deità, dal dio della luce (deus lucis) e dal dio delle tenebre (deus tenebrarum $)^{21}$. I patarini bosniaci erano perseguitati sia dalla chiesa ortodossa che da quella cattolica. Nel secolo XIII il papato tentava a rendere più forte il proprio influsso in Bosnia. Sappiamo, per esempio, che nel $1291 \mathrm{ci}$ furono inviati due francescani che conoscevano la lingua slava. Però l'influsso del capo della cristianità occidentale era allora limitato alle regioni sttentrionali della Bosnia confinanticon l'Ungheria.

E un fatto molto più importante che nel secolo XIII la Bulgaria e la Serbia oscillarono tra l'Oriente e l'Occidente. Accettando a Roma la corona nel 1204, l'imperatore Kalojan acconsenti all'unione con la chiesa romana. Intanto, come nell'Impero bizantino, l'unione non attecchi neanche in Bulgaria e già nel 1235, col permesso dei patriarchi orientali, venne rinnovato il patriarcato ortodosso bulgaro ${ }^{22}$.

19 Sulla politica occidentale dell'imperatore Michele VIII Paleologo: D. Deanakoplos, Emperor Micbael Palaeologus and the West 1258-1282, Hamdem Connect. 1973.

${ }_{20}$ L'unione di Lione, naturalmente, venne rigettata anche dai monaci di Athos. M. Zivojinovic, «Sveta Gora i Lionska unija» (Athos e l'Unione di Lione), Zbornik radova Vizantoloskog instituta u Beograd, 18, 1978, pp. 141-153.

21 S. CIRKOviC, Istorija srednjovekovne bosanske drzave (La storia dello stato bosniaco medievale), Beograd 1964, 101-112.

22 V. VAsIL EVSKUU, "Obnovlenije Bolgarskogo Patriarsestva pri care foanne Asene II v $1235 \mathrm{~g}$ " (Restaurazione del Patriarcato bulgaro sotto 
Nella storia della chiesa serba il secolo XIII ha un' importanza ancora maggiore. Chiedendo il diadema regale al papa, il Gran zupan serbo Stefano chiamava il papa "suo padre spirituale». Dopol'incoronazione, nel 1220 egli chiese al papa di benedirlo, confermandogli, da figlio della chiesa romana, la propria fedeltà ${ }^{23}$. Però ancora più importante è quello che aveva ottenuto, però dall'altra parte, suo fratello Sava, il quale da giovane era fuggito dalla corte paterna per vivere come monaco sul Monte Atos. Nel, approfittando di certo, almeno indirettamente, del crollo dell'Impero bizantino, egli riusei a ottenere a Nicea l' indipendenza della chiesa serba che fino allora era stata subordinata all'arcivescovato di Ocrida. A Nicea lo stesso Sava fu consacrato il primo arcivescovo della chiesa serba indipendente. Questo atto non era importante solamente per la chiesa ma anche per lo stato. Con esso fu consolidata l'indipendenza del giovane regno serbo e in tal modo, è lecito dirlo, la chiesa serba è rimasta definitivamente nella famiglia delle cominità cristiane orientali. Già nel secolo XIV essa ottenne il rango di patriarcato.

In Bulgaria e in Serbia l'influsso del papa si fece sentire più forte all'inizio del secolo XIII, al tempo del papato di Innocenzo III, a ella fine dello stesso secolo, al tempo della politica antibizantina di Carlo I d'Angiò. Il contatto delle due chiese cristiane nei paesi slavi balcanici era un contatto da nemici. Tuttavia ce ne furono degli esempi di collaborazione. Per esempio l'attività di Elena, la moglie del re serbo Stefano Uros I proveniente dalla casa d'Angiò. Di nascita era cattolica e lo restò fino alla fine della sua vita, però nonostante ciò essa era rispettata dalla chiesa serba ortodosa. Nelle regioni littorali dello stato serbo, abitate dalla popolazione cattolica, Elena fece costruire chiese caitoliche e nell'interno del paese dei monasteri ortodossi ${ }^{24}$.

l'Imperatore Ivan Asen II nel 1235) Zxurnal Ministarstva Narodnogo Prosvescenija, 238, 1885, pp. 1-56.

${ }_{23}$ Esistono notizie secondo cui la moglie di Stefano, una veneziana di nascita, avrebbe influito sul marito di aderire alla chiesa occidentale. K. JIRECEK, Istorija Srba, I, p, 169.

24 G. Ostrogorski, op. cit, p. 399. 
Considerato tutto il sopraesposto, possiamo concludere che nel secolo XIII $\mathrm{i}$ contatti della cultura e della civiltà orientali e quelle occidentali nel territorio della penisola balcanica furono intensissimi. Quanto alla politica, la penisola era frantumata in parecchie unità statali delle quali alcune erano latine per conseguenze dirette della quarta crociata. Pertanto tali fondazioni latine erano un corpo estraneo piuttosto rigettatto che accolto dall'organismo balcanico, quindi durante il secolo XIII esse per lo più decaddero. Pare che l'influsso dell'Occidente sull'economia, sebbene meno apparente, fosse più duraturo. Era il tempo di una sempre più rigogliosa fioritura della vita economica in Occidente, mentre l'economia dell'Impero bizantino stava già decadendo per sparire prima del crollo definitivo politico dell'Impero cristiano d'Oriente. I tentativi di reconciliare nel campo spirituale l'Occidente e l'Oriente fatti nel secolo XIII fallirono. L'unione delle due chiese venne spesso discussa. Persino fu proclamata una volta, però mai realizzata. I suoi fautori nell'Impero bizantino erano gli imperatori. Essi non volevano conseguire l'unione per ideali cristiani universali, ma per ragioni prettamente politiche. Per gli stessi motivi i regnanti serbi e bulgari si piegavano saltuariamente a Roma. Tuttavia l'unione non potè attecchire presso la popolazione balcanica ortodossa. In tal modo le conquiste latine della penisola balcanica nel secolo XIII non attenuarono bensi acuirono il contrasto fra l'Oriente e l'Occidente. 